\title{
USO DO PROCEDIMENTO DE MÁSCARA PARA VERIFICAR RELAÇÕES DE CONTROLE DE ESTÍMULOS EM MACACOS-PREGO
}

\author{
USE OF MASK PROCEDURE TO VERIFY STIMULUS CONTROL RELATIONS IN \\ CAPUCHIN MONKEYS
}

\author{
Ilara Reis Nogueira da Cruz, Carlos Rafael Fernandes Picanço e \\ Romariz da Silva Barros ${ }^{1}$ \\ UNIVERSIDADE FEDERAL DO PARÁ
}

\begin{abstract}
RESUMO
Diferenças sutis entre as relações de controle planejadas pelo experimentador e aquelas efetivamente estabelecidas nos treinos discriminativos são frequentemente encontradas em estudos com participantes humanos com desenvolvimento severamente atrasado ou com sujeitos não humanos. Grande parte das vezes, essas diferenças só são identificadas por meio de testes. O presente estudo avaliou a viabilidade do uso do procedimento de máscara para verificar relações de controle em discriminação condicional (emparelhamento ao modelo por identidade) com macacos. Foram utilizados dois macacos da espécie Cebus cf. apella como sujeitos. O procedimento consistiu no treino de emparelhamento ao modelo por identidade e testes de relações de controle, nos quais ora o $\mathrm{S}+$, ora o $\mathrm{S}-$ era substituído por uma janela vazia (máscara). Com a remoção desses estímulos em tentativas de sonda, avaliou-se sua participação na determinação de diferentes tipos de relações de controle do comportamento de escolha: Controle pelo S- ou Controle por Rejeição; Controle pelo S+ ou Controle por Seleção; ou ambos, ou seja, Controle Misto. Os dados obtidos mostram que (1) as discriminações estabelecidas não deterioraram com a aplicação dos testes e (2) as escolhas nas tentativas de sonda permitiram inferir com sucesso as relações de controle vigentes. O sujeito M09 apresentou Controle por Rejeição inicialmente em uma das relações condicionais e Controle Misto nas outras relações testadas. O sujeito M16 apresentou Controle Misto em todas as relações testadas. Com esses achados, o procedimento de máscara pode ser considerado adequado para verificação de relações de controle em discriminações condicionais com macacos como sujeitos. Encoraja-se o uso desse procedimento em estudos futuros sobre classes de equivalência com não humanos para verificar se de fato foram estabelecidas relações condicionais arbitrárias modelo-S+ (i.e., Controle por Seleção ou Misto).
\end{abstract}

Palavras-chave: procedimento de máscara, emparelhamento ao modelo por identidade, Cebus cf. apella.

\begin{abstract}
Subtle differences between the stimulus control relations that are programmed by the experimenter and those effectively established in discriminative training are frequently found in non-human subjects or human participants with severe developmental disabilities. Most of the times, such differences are only identified through testing procedures. The present study evaluated the viability of using mask procedure to verify stimulus control relations in conditional discriminations (identity matching to sample) with monkeys as subjects. Two capuchin monkeys (Cebus of. apella) participated. The procedure comprised identity matching to sample training and tests for stimulus control relations with a blank comparison (mask) replacing sometimes S+ sometimes S-. By selectively removing those stimuli in probe trails, we evaluated their participation in determining different types of controlling relations: Control by S- or Reject Control; Control by S+ or Select Control, or both, that is, Mixed Control. Results show that (1) baseline discriminations did not deteriorate during the tests and (2) choice responses in probe trials let successfully infer the on-going stimulus control relations. Subject M09 presented initially Reject Control for one of the tested relations and Mixed Control for the other relations. Subject M16 presented Mixed Control for all tested relations. The findings suggest that mask procedure is adequate to evaluate stimulus control in conditional relations with monkeys. We encourage its use in future studies on equivalence class formation with non-humans to verify if in fact the arbitrary Sample-S+ conditional relations (i.e., Select or Mixed control) were established.
\end{abstract}

Keywords: mask procedure, identity matching, Cebus of. apella.

Um dos desafios da pesquisa sobre controle de estímulos complexo com sujeitos não humanos ou com participantes humanos com desenvolvimento severamente atrasado é que frequentemente as relações de controle efetivamente estabelecidas nos treinos

\footnotetext{
${ }^{1}$ Endereço para correspondência: Romariz Barros - Rod.Arthur Bernardes, 1.650, Q6 L15 - Belém/PA - CEP: 66825-000 - Tels.: (91) 32489004 / 8800-9004. E-mail: romarizsb@gmail.com. Financiamento: CNPq/INCT-ECCE (BRASIL) e NIH (USA).
} 
discriminativos são sutilmente diferentes das relações de controle planejadas pelo experimentador (Barros, Galvão, Brino, Goulart, \& McIlvane, 2005).

Por exemplo, o controle não programado pela posição dos estímulos foi relatado por Iversen, Sidman e Carrigan (1986 - ver, também, Iversen, 1997; Lionello \& Urcuioli, 1998). Nesses estudos, o emparelhamento ao modelo por identidade era treinado com o estímulo modelo sendo apresentado em uma janela central (entre três janelas alinhadas horizontalmente), e os estímulos de comparação, nas duas janelas laterais. Uma vez aprendidas as discriminações, os experimentadores passavam a apresentar os estímulos modelo em quaisquer das três janelas, e os estímulos de comparação, nas duas janelas remanescentes. A mudança na posição dos estímulos produziu a deterioração das discriminações, mostrando que as relações de controle efetivamente estabelecidas no treino incluíam as posições dos estímulos e, portanto, não consistiam no verdadeiro emparelhamento ao modelo por identidade planejado pelos experimentadores.

Um tipo de digressão de relações de controle ainda mais sutil foi relatado por Sidman (1987). O autor identificou que dois tipos de relações de controle podem ser inferidos quando um participante mostra precisão em uma tarefa discriminativa (como o emparelhamento ao modelo por identidade exemplificado): o Controle por Seleção e o Controle por Rejeição. O Controle por Seleção, ou Controle por $\mathrm{S}+$, consiste na escolha do estímulo S+ sob controle das propriedades desse estímulo; o Controle por Rejeição, ou Controle por S-, consiste na escolhe do S+ sob controle das propriedades do S-, ou seja, por rejeição ao S-.
O problema de se obterem desempenhos controlados por rejeição, quando se planeja Controle por Seleção no procedimento de emparelhamento ao modelo, por exemplo, é que não se estabelecem as relações planejadas entre modelo e comparação correto $(\mathrm{S}+)$. Ao invés disso, estabelecem-se relações entre o modelo e a comparação S-. Isso pode, por exemplo, determinar resultados negativos em testes de propriedades de simetria ou transitividade em estudos sobre a formação de classes de equivalência (Sidman \& Tailby, 1982).

Isso pode explicar pelo menos parte dos fracassos obtidos nos testes das propriedades comprobatórias da equivalência de estímulos com sujeitos não humanos e com participantes humanos com desenvolvimento severamente atrasado. A Teoria da Coerência de Topografia de Controle de Estímulos, cuja precursora foi Ray (1969), foi retomada e estendida por Mcllvane e Dube (2003 - ver, também, Dube \& Mcllvane, 1996; Mcllvane \& Dube, 1992), mostrando como podemos relacionar os fracassos no estabelecimento de classes de equivalência com a especificação inadequada das topografias de controle de estímulos do comportamento do sujeito. Essa teoria mostra que, nos estudos sobre a formação de classes de equivalência por via de procedimento de emparelhamento ao modelo, quando as relações modelo-S+ não são estabelecidas, conforme planejado pelo experimentador, é impossível ter sucesso nos testes das propriedades de equivalência dessas supostas relações, simplesmente porque elas não existem. As relações verdadeiramente estabelecidas são outras, como, por exemplo, o Controle por Rejeição. Esse tipo de fracasso nos testes (possivelmente comum entre os 
casos de fracassos com sujeitos não humanos) não põe em questionamento teorias sobre a formação de classes de equivalência, simplesmente porque nesses casos não há relações de equivalência sob teste, mas, sim, outras relações não planejadas pelo experimentador.

Questões como essa mostram a importância de desenvolver e avaliar procedimentos para verificar as relações de controle em discriminações condicionais no procedimento de emparelhamento ao modelo.

Para verificar as relações de controle estabelecidas em treinos de relações condicionais em estudos com participantes humanos com desenvolvimento severamente atrasado, Mcllvane, Kledaras, Munson, King, de Rose e Stoddard (1987) desenvolveram um procedimento que chamaram de blank comparison procedure (procedimento de comparação vazio), ou mask procedure (procedimento de máscara). Nesse procedimento, a máscara (MK) substitui em igual número de vezes ora o estímulo de comparação positivo $(\mathrm{S}+)$, ora o estímulo de comparação negativo (S-). A partir daí, pode-se verificar se o treino aplicado foi eficaz no estabelecimento dos dois tipos de relação de controle no repertório treinado. Se a omissão do estímulo de comparação positivo $(\mathrm{S}+)$ produz queda da precisão do desempenho, considera-se esse fato como uma evidência de Controle por Seleção. Se a omissão do estímulo de comparação negativo (S-) produz queda da precisão do desempenho, considera-se esse fato como uma evidência de Controle por Rejeição. Registrado um desempenho preciso tanto nas tentativas com omissão do estímulo comparação positivo quanto do negativo, o Controle Misto é documentado, ou seja, controle tanto por rejeição quanto por seleção.
Nessa direção, Goulart, Mendonça, Barros, Galvão e Mcllvane (2005) conduziram um estudo utilizando pela primeira vez o procedimento de máscaras com macacos-prego. O principal objetivo do estudo foi avaliar a viabilidade do uso do procedimento de máscara para avaliar relações de controle especificamente em discriminações simples com macacos como sujeito.

No primeiro experimento do referido estudo, inicialmente foi efetuado o treino de uma discriminação simples simultânea entre dois estímulos (A1 e A2), com A1 funcionando como S+, e A2, como S-. Quando os sujeitos alcançaram um critério de acertos $\geq$ ("maior ou igual a") 90\%, as funções discriminativas dos estímulos foram revertidas: A2 passou a funcionar como $\mathrm{S}+$, e A1, como S-. Esse procedimento de reversões das discriminações foi repetido sucessivamente. Após a aquisição rápida das reversões ter sido demonstrada, foram apresentados os testes com o procedimento de máscara. Esses testes consistiram na apresentação de tentativas de sonda (em meio a tentativas de linha de base), nas quais ora o $\mathrm{S}+$, ora o $\mathrm{S}-$ era substituído pela máscara. Foram documentados comportamentos consistentes com Controle por Seleção em alguns casos e Controle por Rejeição em outros, mas houve considerável variabilidade.

Com o objetivo de reduzir a variabilidade nos dados, foi conduzido o Experimento II, no qual o procedimento de máscara foi usado para estabelecer diretamente, por meio de treino, as relações de Controle por Seleção e por Rejeição. Nesse caso, o procedimento de máscara passou a ser utilizado não mais como um procedimento de avaliação das relações de controle, mas como um procedimento de 
treino. Havia três tipos de tentativas: "A1+/ A2-" (ambos os estímulos discriminativos eram apresentados); "A1+/MK-" (a máscara substituía o S-); e "MK+/A2-" (a máscara substituía o $\mathrm{S}+$ ). Para atingir alta precisão de desempenho nessas condições, os sujeitos tinham de aprender não apenas a selecionar o $\mathrm{S}+$, mas também rejeitar o $\mathrm{S}-$, nesse caso respondendo na máscara.

Após obtenção de desempenho preciso no treino com máscaras descrito, testes de relações de controle foram conduzidos. Nesse caso, as máscaras foram removidas, e estímulos completamente novos para os sujeitos substituíam ora o $\mathrm{S}+$, ora o $\mathrm{S}-$ em tentativas de sonda. Esses estímulos desempenhavam, portanto, o mesmo papel que as máscaras desempenharam no Experimento I. Os novos testes mostraram de maneira consistente tanto Controle por Seleção quanto Controle por Rejeição.

Esse estudo mostrou que o procedimento de máscara pode ser útil para avaliar relações de controle com macacos como sujeitos no contexto de discriminações simples. O estudo mostrou, também, que, quando as relações de controle planejadas não parecem consistentes nos testes, esse mesmo procedimento de máscara pode ser usado como procedimento remediativo. Apesar dessas contribuições, o estudo de Goulart et al. (2005) não explorou o uso do procedimento de máscara em discriminações condicionais com macacos.

De fato, ainda não foram relatados estudos que mostrem a eficácia do procedimento de máscara para avaliar relações de controle em discriminações condicionais em sujeitos não humanos. A importância desse tipo de estudo está na definição de um procedimento seguro para a investigação da coerência de controle de estímulos em treinos de discriminações condicionais, como nos estudos sobre a formação de classes de equivalência em animais.

O presente estudo explorou essa possibilidade, utilizando macacos-prego como sujeitos. O objetivo foi avaliar a viabilidade do uso do procedimento de máscara para verificar relações de controle no contexto de discriminações condicionais (treino de emparelhamento ao modelo por identidade) com macacos. Verificou-se se, durante um treino de emparelhamento ao modelo por identidade, estavam sendo desenvolvidas as relações de controle necessárias para o real desempenho de emparelhamento ao modelo.

\section{MÉtodo}

\section{Sujeitos}

Foram utilizados dois macacos-prego adultos jovens: Guga (M09) e Bongo (M16). Os dois sujeitos tinham história experimental em treino de mudanças repetidas de discriminações simples, emparelhamento ao modelo por identidade e testes de identidade generalizada. Essa experiência dos sujeitos com discriminações condicionais por identidade não interfere no objetivo do presente estudo, uma vez que se pretendeu, aqui, avaliar a eficácia do procedimento de máscaras para detectar as relações de controle vigentes nessas discriminações condicionais, e não sua eficácia para estabelecê-las. Assim, o fato de os sujeitos terem experiência com emparelhamento ao modelo por identidade apenas acelerou a consecução dos testes para se conhecer quais relações de controle estavam presentes nas relações condicionais estabelecidas por aquele procedimento. 
Cada um dos sujeitos era alojado em uma gaiola-viveiro de $2,5 \times 2,5 \times 2,5 \mathrm{~m}$ junto com outros três animais da mesma espécie. Eles tinham livre acesso a água e eram alimentados uma vez ao dia. A dieta consistia em uma variedade de frutas, legumes, raízes, castanhas, além de ovos, leite, complementos vitamínicos e ração. As condições de alojamento, cuidados veterinários e manejo dos animais, bem como os procedimentos experimentais aqui descritos, foram aprovados junto ao Comitê de Ética em Pesquisa com Animais do Instituto de Ciências Biológicas da Universidade Federal do Pará. O biotério no qual os animais foram mantidos é um criadouro e foi autorizado pelo Ibama para fins científicos.

\section{Equipamento e estímulos}

Foi utilizada uma câmara experimental medindo $0,80 \times 0,80 \times 0,70 \mathrm{~m}$. Na parede frontal da câmara experimental, havia uma janela de 0,26 ×0,26 m, na qual estava acoplado um monitor de tela sensível ao toque, por meio do qual eram apresentados os estí- mulos e registradas as respostas. $\mathrm{O}$ assoalho, o teto e a parede lateral esquerda da câmara experimental foram construídos de tela de aço tipo moeda. Na parede lateral esquerda, localizava-se uma porta de 0,35 × 0,20 m, que funcionava como entrada e saída dos sujeitos.

Acoplado à câmara experimental, encontrava-se um microcomputador AMD K6 150. As sessões eram programadas em um software intitulado EAM 4.0.04 (desenvolvido por Dráusio Capobianco, com financiamento do CNPq), para uso em experimentos envolvendo o treino de relações entre estímulos. Um dispensador automático de pelotas de açúcar sabor banana de $190 \mathrm{mg}$ foi utilizado para consequenciar as respostas consideradas pelo experimentador como corretas.

Os estímulos utilizados eram formas abstratas desenhadas em preto sobre fundo branco quadrado $(5 \times 5 \mathrm{~cm})$, e, em algumas ocasiões, conforme descrito a seguir, apenas o fundo branco era apresentado, funcionando como o que é chamado de máscara, ou, mais propriamente, comparação vazio.

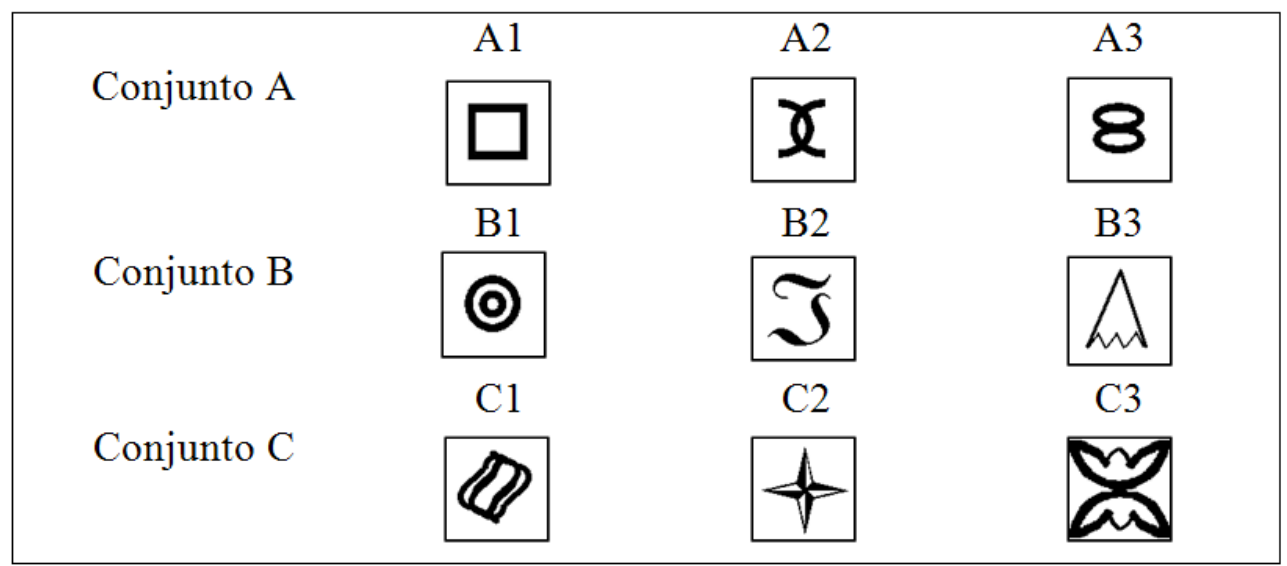

Figura 1. Estímulos utilizados no treino de emparelhamento ao modelo por identidade com os Conjuntos A, B e C. 
A seguir, nas Figuras 1 e 2, podem-se visualizar os três conjuntos de estímulos utilizados ao longo do treino de emparelhamento ao modelo por identidade com os dois sujeitos, bem como suas combinações com a máscara.

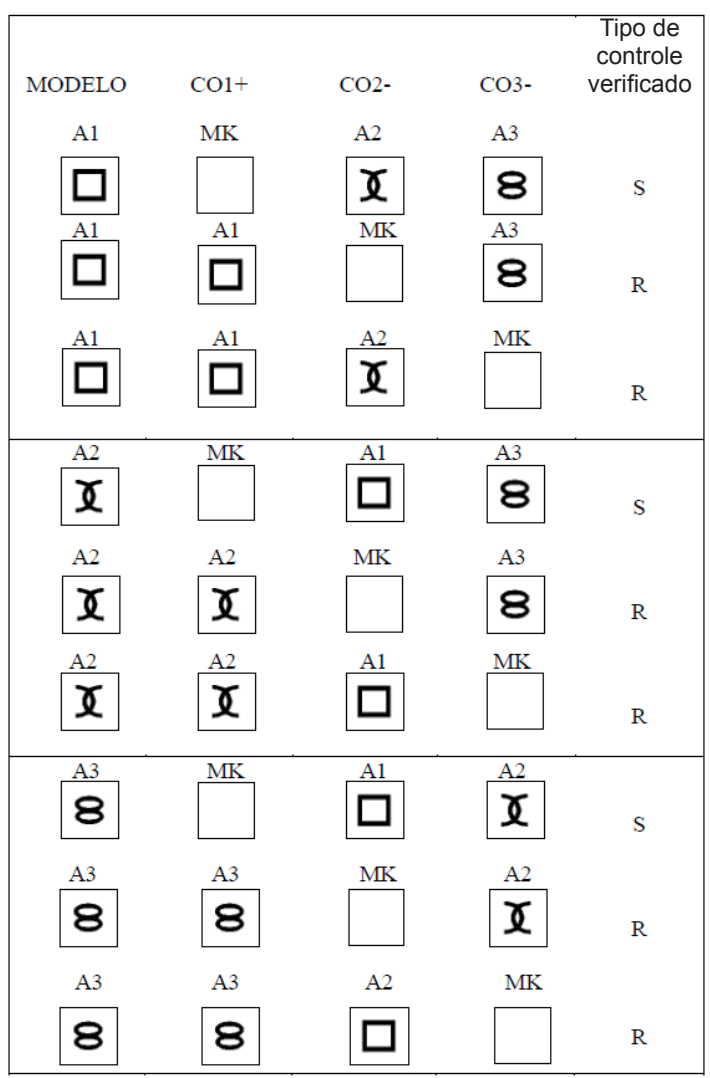

Figura 2. Tipos de tentativas utilizadas nos testes de relações de controle com a utilização do procedimento de máscara (MK) com os sujeitos M09 e M16. São apresentados as relações testadas e o tipo de controle verificado: " $\mathrm{S}$ " indica Controle por Seleção, "R" indica Controle por Rejeição. $\mathrm{CO} 1+=$ Comparação1-S+; CO2- = Comparação2-S-; CO3- = Comparação3-S-.

\section{Procedimento}

Os dois sujeitos foram inicialmente submetidos a um treino de emparelhamento ao modelo por identidade com três conjuntos de três estímulos cada. Em todo o experimento, foi utilizado o procedimento de emparelhamento ao modelo com atraso zero, ou seja, após a resposta de observação ao modelo, este era removido e os estímulos de comparação eram imediatamente apresentados. Cada sessão era composta por 48 tentativas, sendo 16 para cada relação (A1A1, A2A2 e A3A3). Cada tentativa se iniciava com a apresentação de um estímulo modelo (por exemplo, A1). Respostas de tocar esse estímulo produziam sua retirada e a apresentação dos três estímulos de comparação (A1, A2 e A3). Respostas de tocar o estímulo de comparação idêntico ao modelo (ou seja, o $\mathrm{S}+$ ) eram consideradas corretas e produziam o acionamento do comedouro e um intervalo entre tentativas (IET) de seis segundos. Respostas a quaisquer dos estímulos diferentes do modelo (S-) eram consideradas erros e produziam o encerramento da tentativa e o IET (intervalo entre tentativas), sem o acionamento do comedouro. O critério de aquisição das discriminações estabelecido era de obtenção de número de acertos $\geq 90 \%$ do total de tentativas de uma sessão.

Após a aquisição das discriminações condicionais descritas, foram treinadas as discriminações com os estímulos dos Conjuntos B (B1B1, B2B2 e B3B3) e C (C1C1, C2C2 e C3C3) seguindo o mesmo procedimento.

Durante todo o experimento, respostas a A1, B1 e C1 (quando estes funcionavam como $\mathrm{S}+$ ) eram consequenciadas com R1 (Reforço 1, R1); respostas a A2, B2 e C2 (quando funcionavam como $\mathrm{S}+$ ) produziam R2; e respostas a A3, B3 e C3 (quando funcionavam como $\mathrm{S}+$ ) eram consequenciadas com R3. 
Logo em seguida a alcançar o critério no treino de identidade ao modelo com cada um dos conjuntos de estímulos, os sujeitos eram expostos ao teste de relação de controle.

Para a realização dos testes de relações de controle, foi utilizado o procedimento de máscara (MK), ou comparação vazio (McIlvane et al., 1987). Eram apresentados blocos de 48 tentativas, sendo 12 idênticas à linha de base anteriormente descrita e 36 tentativas com máscara. Dentre as 36 tentativas com máscara, 12 eram do tipo A1A1, 12 eram do tipo A2A2 e as demais eram do tipo A3A3. Essa mesma proporção de tentativas foi adotada nos testes com os estímulos dos outros dois conjuntos. Para cada tipo de relação, a máscara substituiu (de maneira imprevisível para o sujeito) oito vezes o estímulo positivo e quatro vezes cada um dos dois estímulos negativos, de modo que ela aparecia igual número de vezes (oito) com a função positiva e com a função negativa. Durante o teste, todas as escolhas corretas eram seguidas de reforço. Esse procedimento foi adotado com o objetivo de evitar os efeitos de deterioração da precisão do desempenho já observado em testes múltiplos em extinção com macacos-prego (Brino, Galvão, \& Barros, 2009). Para garantir a fidedignidade do teste, contudo, a primeira tentativa de cada relação testada (que, portanto, ocorria antes de qualquer reforçamento) assumiu importância crítica: para determinada relação de controle ser postulada, era preciso haver acerto na primeira tentativa em que era testada e em mais duas das três tentativas subsequentes do mesmo tipo (ver, por exemplo, Schusterman \& Kastak, 1993).
A fase seguinte do treino se iniciava caso relações de Controle Misto (Controle por Seleção e por Rejeição em uma mesma tentativa) fossem encontradas. O critério para que o Controle Misto estivesse estabelecido era de que o sujeito acertasse três das quatro tentativas de cada relação verificada e que não errasse na primeira tentativa também de cada relação.

\section{Resultados}

No treino de emparelhamento ao modelo por identidade, o sujeito M09 apresentou desempenho entre 86 e 94\% de acertos em três sessões, alcançando o critério estabelecido. $\mathrm{O}$ treino com os estímulos do Conjunto B foi concluído em duas sessões, com 88 e 97\% de escolhas corretas do sujeito. Quando o mesmo sujeito foi exposto ao treino de identidade com os estímulos do Conjunto C, alcançou o critério em uma única sessão.

Em relação ao sujeito M16, o desempenho apresentado foi entre 55 e 100\% de acertos em quatro sessões de emparelhamento ao modelo por identidade, alcançando, assim, o critério estipulado. $\mathrm{O}$ treino com os estímulos do Conjunto B foi concluído em uma única sessão, com desempenho de 97\%. Quando exposto ao treino de identidade com os estímulos do Conjunto C, o sujeito precisou de uma única sessão para alcançar o critério de precisão, com 94 \% de acertos.

Assim que os sujeitos alcançavam o critério em cada um dos conjuntos de estímulos treinados, eram submetidos aos testes de relações de controle. O desempenho apresentado pelos sujeitos nesses testes está descrito nas tabelas a seguir, correspondentes a cada um dos treinos. 
Tabela 1

Desempenho dos sujeitos M09 e M16 na sessão de teste de relações de controle nas relações de identidade AA. Para cada sujeito, são apresentados, por relação, o percentual de acerto e as respostas de escolha na sequência em que ocorreram. "C" indica resposta ao S+ (acerto). Respostas a S- são indicadas com a apresentação do S- escolhido em lugar de S+. "S" indica que Controle por Seleção foi encontrado. "R" indica que Controle por Rejeição foi encontrado. "M" indica Controle Misto.

\begin{tabular}{|c|c|c|c|c|c|}
\hline Sujeito & $\begin{array}{l}\text { Tipo de } \\
\text { tentativa }\end{array}$ & $\begin{array}{c}\text { Contingências } \\
(\mathrm{Mod}-\mathrm{S}+, \mathrm{S}-, \mathrm{S}-)\end{array}$ & $\begin{array}{l}\text { Sequência de } \\
\text { escolhas }\end{array}$ & $\begin{array}{l}\% \text { de } \\
\text { acerto }\end{array}$ & $\begin{array}{l}\text { Tipo de } \\
\text { controle }\end{array}$ \\
\hline \multirow{12}{*}{ M09 } & \multirow{3}{*}{$\begin{array}{l}\text { Linha de } \\
\text { base }\end{array}$} & $\mathrm{A} 1-\mathrm{A} 1+, \mathrm{A} 2-, \mathrm{A} 3-$ & $\mathrm{C}, \mathrm{C}, \mathrm{A} 3$ & 66,66 & \\
\hline & & $\mathrm{A} 2-\mathrm{A} 2+, \mathrm{A} 1-, \mathrm{A} 3-$ & $\mathrm{C}, \mathrm{A} 3, \mathrm{~A} 3$ & 33,33 & \\
\hline & & $\mathrm{A} 3-\mathrm{A} 3+, \mathrm{A} 1-, \mathrm{A} 2-$ & $\mathrm{C}, \mathrm{C}, \mathrm{C}$ & 100 & \\
\hline & \multirow{9}{*}{ Teste } & $\mathrm{A} 1-\mathrm{A} 1+, \mathrm{A} 2-, \mathrm{MK}-$ & $\mathrm{C}, \mathrm{C}, \mathrm{MK}, \mathrm{MK}$ & 50 & \multirow{3}{*}{$\mathrm{R}$} \\
\hline & & $\mathrm{A} 1-\mathrm{A} 1+, \mathrm{MK}-, \mathrm{A} 3-$ & $\mathrm{C}, \mathrm{C}, \mathrm{MK}, \mathrm{C}$ & 75 & \\
\hline & & $\mathrm{A} 1-\mathrm{MK}+, \mathrm{A} 2-, \mathrm{A} 3-$ & $\mathrm{C}, \mathrm{C}, \mathrm{C}, \mathrm{C}$ & 100 & \\
\hline & & $\mathrm{A} 2-\mathrm{A} 2+, \mathrm{A} 1-, \mathrm{MK}-$ & $\mathrm{C}, \mathrm{C}, \mathrm{C}, \mathrm{C}$ & 100 & \multirow{3}{*}{ M } \\
\hline & & $\mathrm{A} 2-\mathrm{A} 2+, \mathrm{MK}-, \mathrm{A} 3-$ & $\mathrm{C}, \mathrm{C}, \mathrm{C}, \mathrm{C}$ & 100 & \\
\hline & & $\mathrm{A} 2-\mathrm{MK}+, \mathrm{A} 1-, \mathrm{A} 3-$ & $\mathrm{C}, \mathrm{C}, \mathrm{C}, \mathrm{C}$ & 100 & \\
\hline & & $\mathrm{A} 3-\mathrm{A} 3+, \mathrm{A} 2-, \mathrm{MK}-$ & $\mathrm{C}, \mathrm{C}, \mathrm{C}, \mathrm{C}$ & 100 & \multirow{3}{*}{ M } \\
\hline & & $\mathrm{A} 3-\mathrm{A} 3+, \mathrm{MK}-, \mathrm{A} 2-$ & $\mathrm{C}, \mathrm{C}, \mathrm{C}, \mathrm{C}$ & 100 & \\
\hline & & $\mathrm{A} 3-\mathrm{MK}+, \mathrm{A} 1-, \mathrm{A} 2-$ & $\mathrm{C}, \mathrm{C}, \mathrm{C}, \mathrm{C}$ & 100 & \\
\hline \multirow{12}{*}{ M16 } & \multirow{3}{*}{$\begin{array}{l}\text { Linha de } \\
\text { base }\end{array}$} & $\mathrm{A} 1-\mathrm{A} 1+, \mathrm{A} 2-\mathrm{A} 3-$ & $\mathrm{C}, \mathrm{C}, \mathrm{C}$ & $100 \%$ & \\
\hline & & $\mathrm{A} 2-\mathrm{A} 2+, \mathrm{A} 1-, \mathrm{A} 3-$ & $\mathrm{C}, \mathrm{C}, \mathrm{C}$ & $100 \%$ & \\
\hline & & $\mathrm{A} 3-\mathrm{A} 3+, \mathrm{A} 1-, \mathrm{A} 2-$ & $\mathrm{C}, \mathrm{C}, \mathrm{C}$ & $100 \%$ & \\
\hline & \multirow{9}{*}{ Teste } & $\mathrm{A} 1-\mathrm{A} 1+, \mathrm{A} 2-, \mathrm{MK}-$ & $\mathrm{C}, \mathrm{C}, \mathrm{C}, \mathrm{C}$ & $100 \%$ & \multirow{3}{*}{ M } \\
\hline & & $\mathrm{A} 1-\mathrm{A} 1+, \mathrm{MK}-, \mathrm{A} 3-$ & $\mathrm{C}, \mathrm{C}, \mathrm{C}, \mathrm{C}$ & $100 \%$ & \\
\hline & & $\mathrm{A} 1-\mathrm{MK}+, \mathrm{A} 2-, \mathrm{A} 3-$ & $\mathrm{C}, \mathrm{C}, \mathrm{C}, \mathrm{C}$ & $100 \%$ & \\
\hline & & $\mathrm{A} 2-\mathrm{A} 2+, \mathrm{MK}-, \mathrm{A} 3-$ & $\mathrm{C}, \mathrm{C}, \mathrm{C}, \mathrm{C}$ & $100 \%$ & \multirow{3}{*}{ M } \\
\hline & & $\mathrm{A} 2-\mathrm{A} 2+, \mathrm{A} 1-, \mathrm{MK}-$ & A1, C, C, C & $75 \%$ & \\
\hline & & $\mathrm{A} 2-\mathrm{MK}+, \mathrm{A} 1-, \mathrm{A} 3-$ & $\mathrm{C}, \mathrm{C}, \mathrm{C}, \mathrm{C}$ & $75 \%$ & \\
\hline & & $\mathrm{A} 3-\mathrm{A} 3+, \mathrm{A} 1-, \mathrm{MK}-$ & $\mathrm{C}, \mathrm{C}, \mathrm{C}, \mathrm{C}$ & $100 \%$ & \multirow{3}{*}{ M } \\
\hline & & $\mathrm{A} 3-\mathrm{A} 3+, \mathrm{MK}-, \mathrm{A} 2-$ & $\mathrm{C}, \mathrm{C}, \mathrm{C}, \mathrm{C}$ & $100 \%$ & \\
\hline & & $\mathrm{A} 3-\mathrm{MK}+, \mathrm{A} 1-, \mathrm{A} 2-$ & $\mathrm{C}, \mathrm{C}, \mathrm{C}, \mathrm{C}$ & $75 \%$ & \\
\hline
\end{tabular}




\section{Tabela 2}

Desempenho dos sujeitos M09 e M16 na sessão de teste de relações de controle nas relações de identidade BB. Para cada sujeito, são apresentados, por relação, o percentual de acerto e as respostas de escolha na sequência em que ocorreram. "C" indica resposta ao S+ (acerto).

Respostas a S- são indicadas com a apresentação do S- escolhido em lugar de S+. "S" indica que foi encontrado Controle por Seleção. "R" indica Controle por Rejeição. "M" indica Controle Misto.

\begin{tabular}{|c|c|c|c|c|c|}
\hline Sujeito & $\begin{array}{l}\text { Tipo de } \\
\text { tentativa }\end{array}$ & $\begin{array}{l}\text { Contingências } \\
(\text { Mod-S+, S-, S-) }\end{array}$ & $\begin{array}{l}\text { Sequência de } \\
\text { escolhas }\end{array}$ & $\begin{array}{c}\% \text { de } \\
\text { acerto }\end{array}$ & $\begin{array}{l}\text { Tipo de } \\
\text { controle }\end{array}$ \\
\hline \multirow{12}{*}{ M09 } & \multirow{3}{*}{$\begin{array}{l}\text { Linha de } \\
\text { base }\end{array}$} & $\mathrm{B} 1-\mathrm{B} 1+, \mathrm{B} 2-, \mathrm{B} 3-$ & $\mathrm{C}, \mathrm{C}, \mathrm{C}$ & $100 \%$ & \\
\hline & & $\mathrm{B} 2-\mathrm{B} 2+, \mathrm{B} 1-, \mathrm{B} 3-$ & $\mathrm{C}, \mathrm{C}, \mathrm{C}$ & $100 \%$ & \\
\hline & & $\mathrm{B} 3-\mathrm{B} 3+, \mathrm{B} 1-, \mathrm{B} 2-$ & $\mathrm{C}, \mathrm{C}, \mathrm{C}$ & $100 \%$ & \\
\hline & \multirow{9}{*}{ Teste } & $\mathrm{B} 1-\mathrm{B} 1+, \mathrm{B} 2-\mathrm{MK}-$ & $\mathrm{C}, \mathrm{C}, \mathrm{C}, \mathrm{C}$ & $100 \%$ & \multirow{3}{*}{ M } \\
\hline & & $\mathrm{B} 1-\mathrm{B} 1+, \mathrm{MKB} 3-$ & $\mathrm{C}, \mathrm{C}, \mathrm{C}, \mathrm{C}$ & $100 \%$ & \\
\hline & & $\mathrm{B} 1-\mathrm{MK}+, \mathrm{B} 2-, \mathrm{B} 3-$ & $\mathrm{C}, \mathrm{C}, \mathrm{C}, \mathrm{C}$ & $100 \%$ & \\
\hline & & $\mathrm{B} 2-\mathrm{B} 2+, \mathrm{B} 1-, \mathrm{MK}-$ & $\mathrm{C}, \mathrm{C}, \mathrm{C}, \mathrm{C}$ & $100 \%$ & \multirow{3}{*}{ M } \\
\hline & & $\mathrm{B} 2-\mathrm{B} 2+, \mathrm{MK}-, \mathrm{B} 3-$ & $\mathrm{C}, \mathrm{C}, \mathrm{C}, \mathrm{C}$ & $100 \%$ & \\
\hline & & $\mathrm{B} 2-\mathrm{MK}+, \mathrm{B} 1-, \mathrm{B} 3-$ & $\mathrm{C}, \mathrm{C}, \mathrm{C}, \mathrm{C}$ & $75 \%$ & \\
\hline & & $\mathrm{B} 3-\mathrm{B} 3+, \mathrm{B} 1-, \mathrm{MK}-$ & $\mathrm{C}, \mathrm{C}, \mathrm{C}, \mathrm{C}$ & $100 \%$ & \multirow{3}{*}{ M } \\
\hline & & B3-B3+, MK-, B2- & $\mathrm{C}, \mathrm{C}, \mathrm{C}, \mathrm{C}$ & $100 \%$ & \\
\hline & & B3-MK+, B1-, B2- & $\mathrm{C}, \mathrm{C}, \mathrm{C}, \mathrm{C}$ & $75 \%$ & \\
\hline \multirow{12}{*}{ M16 } & \multirow{3}{*}{$\begin{array}{l}\text { Linha de } \\
\text { base }\end{array}$} & $\mathrm{B} 1-\mathrm{B} 1+, \mathrm{B} 2-, \mathrm{B} 3-$ & $\mathrm{C}, \mathrm{C}, \mathrm{C}$ & $100 \%$ & \multirow{3}{*}{ M } \\
\hline & & $\mathrm{B} 2-\mathrm{B} 2+, \mathrm{B} 1-, \mathrm{B} 3-$ & $\mathrm{C}, \mathrm{C}, \mathrm{C}$ & $100 \%$ & \\
\hline & & $\mathrm{B} 3-\mathrm{B} 3+, \mathrm{B} 1-, \mathrm{B} 2-$ & $\mathrm{C}, \mathrm{C}, \mathrm{C}$ & $100 \%$ & \\
\hline & \multirow{9}{*}{ Teste } & $\mathrm{B} 1-\mathrm{B} 1+, \mathrm{B} 2-, \mathrm{MK}-$ & $\mathrm{C}, \mathrm{C}, \mathrm{C}, \mathrm{C}$ & $100 \%$ & \multirow{3}{*}{ M } \\
\hline & & $\mathrm{B} 1-\mathrm{B} 1+, \mathrm{MK}-, \mathrm{B} 3-$ & $\mathrm{C}, \mathrm{C}, \mathrm{C}, \mathrm{C}$ & $100 \%$ & \\
\hline & & $\mathrm{B} 1-\mathrm{MK}+, \mathrm{B} 2-, \mathrm{B} 3-$ & $\mathrm{C}, \mathrm{C}, \mathrm{C}, \mathrm{C}$ & $100 \%$ & \\
\hline & & $\mathrm{B} 2-\mathrm{B} 2+, \mathrm{B} 1-, \mathrm{MK}-$ & $\mathrm{C}, \mathrm{C}, \mathrm{C}, \mathrm{C}$ & $100 \%$ & \multirow{3}{*}{ M } \\
\hline & & $\mathrm{B} 2-\mathrm{B} 2+, \mathrm{MK}-, \mathrm{B} 3-$ & $\mathrm{C}, \mathrm{C}, \mathrm{C}, \mathrm{C}$ & $100 \%$ & \\
\hline & & $\mathrm{B} 2-\mathrm{MK}+, \mathrm{B} 1-, \mathrm{B} 3-$ & $\mathrm{C}, \mathrm{C}, \mathrm{C}, \mathrm{C}$ & $75 \%$ & \\
\hline & & $\mathrm{B} 3-\mathrm{B} 3+, \mathrm{B} 2-, \mathrm{MK}-$ & C, C, C, C & $100 \%$ & \multirow{3}{*}{$\mathrm{M}$} \\
\hline & & $\mathrm{B} 3-\mathrm{B} 3+, \mathrm{B} 1-, \mathrm{MK}-$ & C, C, C, C & $100 \%$ & \\
\hline & & $\mathrm{B} 3-\mathrm{MK}+, \mathrm{B} 1-, \mathrm{B} 2-$ & $\mathrm{C}, \mathrm{C}, \mathrm{C}, \mathrm{C}$ & $100 \%$ & \\
\hline
\end{tabular}


Tabela 3

Desempenho dos sujeitos M09 e M16 na sessão de teste de relações de controle nas relações de identidade CC. Para cada sujeito, são apresentados, por relação, o percentual de acerto e as respostas de escolha na sequência em que ocorreram. "C" indica resposta ao $\mathrm{S}+$ (acerto).

Respostas a S- são indicadas com a apresentação do S- escolhido em lugar de S+. "S" indica Controle por Seleção. "R” indica Controle por Rejeição. "M” indica Controle Misto.

\begin{tabular}{|c|c|c|c|c|c|}
\hline Sujeito & $\begin{array}{l}\text { Tipo de } \\
\text { tentativa }\end{array}$ & $\begin{array}{l}\text { Contingências } \\
(\mathrm{Mod}-\mathrm{S}+, \mathrm{S}-, \mathrm{S}-)\end{array}$ & $\begin{array}{l}\text { Sequência de } \\
\text { escolhas }\end{array}$ & $\begin{array}{l}\% \text { de } \\
\text { acerto }\end{array}$ & $\begin{array}{l}\text { Tipo de } \\
\text { controle }\end{array}$ \\
\hline \multirow{12}{*}{ M09 } & \multirow{3}{*}{$\begin{array}{l}\text { Linha de } \\
\text { base }\end{array}$} & $\mathrm{C} 1-\mathrm{C} 1+, \mathrm{C} 2-, \mathrm{C} 3-$ & $\mathrm{C}, \mathrm{C}, \mathrm{C}$ & $100 \%$ & \\
\hline & & $\mathrm{C} 2-\mathrm{C} 2+, \mathrm{C} 1-, \mathrm{C} 3-$ & C, C, C & $100 \%$ & \\
\hline & & $\mathrm{C} 3-\mathrm{C} 3+, \mathrm{C} 1-, \mathrm{C} 2-$ & $\mathrm{C}, \mathrm{C}, \mathrm{C}$ & $100 \%$ & \\
\hline & \multirow{9}{*}{ Teste } & $\mathrm{C} 1-\mathrm{C} 1+, \mathrm{C} 2-, \mathrm{MK}-$ & $\mathrm{C}, \mathrm{C}, \mathrm{C}, \mathrm{C}$ & $100 \%$ & \multirow{3}{*}{ M } \\
\hline & & $\mathrm{C} 1-\mathrm{C} 1+, \mathrm{MK}-, \mathrm{C} 3-$ & $\mathrm{C}, \mathrm{C}, \mathrm{C}, \mathrm{C}$ & $100 \%$ & \\
\hline & & $\mathrm{C} 1-\mathrm{MK}+, \mathrm{C} 2-, \mathrm{C} 3-$ & $\mathrm{C}, \mathrm{C}, \mathrm{C}, \mathrm{C}$ & $100 \%$ & \\
\hline & & $\mathrm{C} 2-\mathrm{C} 2+, \mathrm{C} 1-, \mathrm{MK}-$ & $\mathrm{C}, \mathrm{C}, \mathrm{C}, \mathrm{C}$ & $100 \%$ & \multirow{3}{*}{ M } \\
\hline & & $\mathrm{C} 2-\mathrm{C} 2+, \mathrm{MK}-, \mathrm{C} 3-$ & $\mathrm{C}, \mathrm{C}, \mathrm{C}, \mathrm{C}$ & $100 \%$ & \\
\hline & & $\mathrm{C} 2-\mathrm{MK}+, \mathrm{C} 1-, \mathrm{C} 3-$ & $\mathrm{C}, \mathrm{C}, \mathrm{C}, \mathrm{C}$ & $75 \%$ & \\
\hline & & $\mathrm{C} 3-\mathrm{C} 3+, \mathrm{C} 1-, \mathrm{MK}-$ & $\mathrm{C}, \mathrm{C}, \mathrm{C}, \mathrm{C}$ & $100 \%$ & \multirow{3}{*}{ M } \\
\hline & & $\mathrm{C} 3-\mathrm{C} 3+, \mathrm{MK}-, \mathrm{C} 2-$ & $\mathrm{C}, \mathrm{C}, \mathrm{C}, \mathrm{C}$ & $100 \%$ & \\
\hline & & $\mathrm{C} 3-\mathrm{MK}+, \mathrm{C} 1-, \mathrm{C} 2-$ & $\mathrm{C}, \mathrm{C}, \mathrm{C}, \mathrm{C}$ & $75 \%$ & \\
\hline \multirow{12}{*}{ M16 } & \multirow{3}{*}{$\begin{array}{l}\text { Linha de } \\
\text { base }\end{array}$} & $\mathrm{C} 1-\mathrm{C} 1+, \mathrm{C} 2-, \mathrm{C} 3-$ & $\mathrm{C}, \mathrm{C}, \mathrm{C}$ & $100 \%$ & \\
\hline & & $\mathrm{C} 2-\mathrm{C} 2+, \mathrm{C} 1-, \mathrm{C} 3-$ & C, C, C & $100 \%$ & \\
\hline & & $\mathrm{C} 3-\mathrm{C} 3+, \mathrm{C} 1-, \mathrm{C} 2-$ & $\mathrm{C}, \mathrm{C}, \mathrm{C}$ & $100 \%$ & \\
\hline & \multirow{9}{*}{ Teste } & $\mathrm{C} 1-\mathrm{C} 1+, \mathrm{C} 2-, \mathrm{MK}-$ & $\mathrm{C}, \mathrm{C}, \mathrm{C}, \mathrm{C}$ & $100 \%$ & \multirow{3}{*}{ M } \\
\hline & & $\mathrm{C} 1-\mathrm{C} 1+, \mathrm{MK}-, \mathrm{C} 3-$ & $\mathrm{C}, \mathrm{C}, \mathrm{C}, \mathrm{C}$ & $100 \%$ & \\
\hline & & $\mathrm{C} 1-\mathrm{MK}+, \mathrm{C} 2-, \mathrm{C} 3-$ & $\mathrm{C}, \mathrm{C}, \mathrm{C}, \mathrm{C}$ & $100 \%$ & \\
\hline & & $\mathrm{C} 2-\mathrm{C} 2+, \mathrm{C} 1-, \mathrm{MK}-$ & $\mathrm{C}, \mathrm{C}, \mathrm{C}, \mathrm{MK}$ & $75 \%$ & \multirow{3}{*}{ M } \\
\hline & & $\mathrm{C} 2-\mathrm{C} 2+, \mathrm{MK}-, \mathrm{C} 3-$ & $\mathrm{C}, \mathrm{C}, \mathrm{C}, \mathrm{C}$ & $100 \%$ & \\
\hline & & $\mathrm{C} 2-\mathrm{MK}+, \mathrm{C} 1-, \mathrm{C} 3-$ & $\mathrm{C}, \mathrm{C}, \mathrm{C}, \mathrm{C}$ & $100 \%$ & \\
\hline & & $\mathrm{C} 3-\mathrm{C} 3+, \mathrm{C} 1-, \mathrm{MK}-$ & $\mathrm{C}, \mathrm{C}, \mathrm{C}, \mathrm{MK}$ & $75 \%$ & \multirow{3}{*}{ M } \\
\hline & & $\mathrm{C} 3-\mathrm{C} 3+, \mathrm{MK}-, \mathrm{C} 2-$ & $\mathrm{C}, \mathrm{C}, \mathrm{C}, \mathrm{C}$ & $100 \%$ & \\
\hline & & C3-MK+, C1-, C2- & $\mathrm{C}, \mathrm{C}, \mathrm{C}, \mathrm{C}$ & $100 \%$ & \\
\hline
\end{tabular}


Como pôde ser observado nas tabelas com resultados dos testes, o sujeito M09 apresentou Controle por Rejeição em um momento inicial dos testes, respondendo à máscara quando esta substituía um dos estímulos de comparação negativo em três das tentativas na relação A1A1. Contudo, o sujeito M09 apresentou Controle Misto em todas as outras relações testadas com os estímulos do mesmo conjunto e dos outros conjuntos (B e C).

No que concerne ao sujeito M16, este apresentou Controle Misto em todas as relações testadas, demonstrando que o treino havia estabelecido um repertório verdadeiro de emparelhamento ao modelo por identidade, de acordo com o planejamento experimental. Com ambos os sujeitos, o repertório de linha de base manteve-se consistente durante a maioria das sessões de teste de relações de controle, o que evidencia que os testes causaram pouca ou nenhuma deterioração nas relações estabelecidas ao longo do treino.

\section{DisCUSSÃO}

O treino de emparelhamento ao modelo empregado neste estudo viabilizou que os sujeitos apresentassem desempenho preciso na linha de base e nas tentativas de sonda; contudo, M09, ao ser submetido ao teste de relações de controle com os estímulos do Conjunto A (relação A1A1), apresentou Controle por Rejeição, respondendo três vezes à máscara quando esta substituía um dos estímulos de comparação negativos. Chama a atenção o fato de que Controle por Rejeição foi encontrado com M09 apenas no primeiro teste com a máscara. $\mathrm{O}$ fato de a máscara não ter sido introduzida ao longo de um pré-teste (fase preparatória para o teste com apresentação da máscara ao sujeito para contornar possíveis efeitos de novidade) pode ter contribuído para o surgimento de alguma "preferência" de escolha do sujeito pela máscara. Nos testes subsequentes com os outros conjuntos e até mesmo com tentativas de outras relações AA, o sujeito apresentou desempenho consistente com as contingências programadas.

No estudo de Goulart et al. (2005), os sujeitos também, inicialmente, apresentaram deterioração no desempenho com a introdução da máscara, e, posteriormente, no segundo experimento do estudo, no qual a máscara ou a introdução de estímulos novos já não representavam uma novidade, os mesmos sujeitos mostraram desempenho consistente.

Quanto ao sujeito M16, este apresentou Controle Misto em todos os testes empregados e manteve sua linha de base com a mesma precisão que apresentava durante o treino.

Mesmo com o desempenho inconsistente de M09 diante de uma das nove relações testadas, o panorama geral do desempenho dos dois sujeitos refletiu um bom estabelecimento de relações de controle de estímulos, o que é dificilmente obtido com sujeitos não humanos.

A utilização do procedimento de máscara mostrou-se uma estratégia de grande utilidade na verificação do estabelecimento dessas relações de controle durante o treino empregado, uma vez que torna possível que ao longo do treino se possa adequar o procedimento de forma mais efetiva, caso seja detectado que o desempenho dos sujeitos não está sendo compatível com as relações de controle de estímulos planejadas pelo experimentador. 
Os achados aqui relatados também confirmam as possibilidades levantadas pelo estudo de Goulart et al. (2005), que indicaram a possibilidade de uso bem-sucedido do procedimento de máscara para identificar relações de controle em discriminações condicionais com macacos como sujeitos. Encoraja-se o uso desse procedimento em estudos futuros sobre classes de equivalência com não humanos para verificar se de fato foram estabelecidas relações condicionais arbitrárias modelo-S+ (i.e., Controle por Seleção ou Misto).

\section{REFERÊNCIAS}

Barros, R. S., Galvão, O. F., Brino, A. L. F., Goulart, P. K. R., \& Mcllvane, W. J. (2005).Variáveis de procedimento na pesquisa sobre classes de equivalência: contribuições para o estudo do comportamento simbólico. Revista Brasileira de Análise do Comportamento, 1, 15-25.

Brino, A. L. F., Galvão, O. F., \& Barros, R. S. (2009). Successive identity matching to sample tests without reinforcement in Cebus apella. Ciências \& Cognição, 14, 2-11.

Dube, W.V., \& Mcllvane, W.J. (1996). Some implications of a stimulus control topography analysis for emergent stimulus classes. In T. R. Zentall \& P. M. Smeets (Eds.). Stimulus class formation in humans and animals (pp. 197-218). Amsterdam, NL: Elsevier North Holland.

Goulart, P. R. K., Mendonça, M. B., Barros, R. S., Galvão, O. F., \& McIlvane, W. J. (2005). A note on select- and reject-controlling relations in the simple discrimination of capuchin monkeys (Cebus apella). Behavioural Processes, 69, 295-302.
Iversen, I. H. (1997). Matching-to-sample performance in rats: A case of mistake identity? Journal of the Experimental Analysis of Behavior, 68, 27-45.

Iversen, I. H., Sidman, M., \& Carrigan, P. (1986). Stimulus definition in conditional discriminations. Journal of the Experimental Analysis of Behavior, 45, 297-304.

Lionello, K. M., \& Urcuioli, P. J. (1998). Control by location in pigeons matching-to-sample. Journal of the Experimental Analysis of Behavior, 70, 235251.

Mcllvane, W. J., \& Dube, W.V. (1992). Stimulus control shaping and stimulus control topographies. The Behavior Analyst, 15, 89-94.

McIlvane, W. J., \& Dube, W. V. (2003). Stimulus control topography coherence theory: Foundations and extensions. The Behavior Analyst, 26, 195-213.

Mcllvane, W. J., Kledaras, J. B., Munson, L. C., King, K. A., de Rose, J. C., \& Stoddard, L. T. (1987). Controlling relations in conditional discrimination and matching by exclusion. Journal of the Experimental Analysis of Behavior, 48, 187-208.

Ray, B. A. (1969). Selective attention: The effects of combining stimuli which control incompatible behavior. Journal of Experimental Analysis of Behavior, 12, 539-550.

Schusterman, R, J., \& Kastak, D. (1993). A california sea lion (Zalophus californianus) is capable of forming equivalence relations. The Psychological Record, 43, 823-839.

Sidman, M. (1987). Two choices are not enough. The Behavior Analyst, 22, 11-18.

Sidman, M., \& Tailby, W. (1982). Conditional discrimination vs. matching to sample: An expansion of the testing paradigm. Journal of the Experimental Analysis of Behavior, 37, 5-22. 